\title{
LA TOMA DE DEGISIONES Y LA GESTIÓN POR OBJETIVOS EN LA EMPRESA PERUANA
}

\author{
DECISION MAKING AND MANAGEMENT BY OBJECTIVES IN PERUVIAN COMPANY
}

\section{José Domingo Begazo Villanueva* d180045@yahoo.es}

[RECEPCIÓN: JULIO DEL 2014/CONFORMIDAD: SETIEMBRE DEL 2014]

\section{RESUMEN}

Al encontrarnos en una sociedad, donde el conocimiento es comoditizado y la toma de decisiones solo es el proceso a través del cual se identifica una necesidad de decisión, estos no son suficientes para establecer alternativas, analizarlas, elegir una de ellas e implementarla para finalmente evaluar los resultados. Se debe complementar con una gestión por objetivos que constituya una forma de conducción y organización de las administraciones que base su concepción en la previsión y preparación anticipada de logros y en la delimitación y asignación de responsabilidades. Es así que, la toma de decisiones es una herramienta que permite hacer las mejores decisiones en beneficio de la empresa, repercutiendo en la gestión, identificando los objetivos que llevarán a lograr las ventajas competitivas y el éxito en el mercado. El problema de la presente investigación se centra en la correlación de la toma de decisiones y la gestión por objetivos en la empresa peruana; buscando afianzar una política de compromisos y responsabilidades por resultados que complemente el paradigma de la gestión por normas y procedimientos predominante en las formas de administración de la empresa peruana.

Palabras claves: Toma de decisiones, gestión por objetivos, empresa.

\begin{abstract}
Since we are in a society where knowledge is commoditized, making decisions only being the process through which a need for a decision is identified, it is not sufficient to establish alternatives, analyze them and choose one of them, and implement it to finally assess the results. Should be supplemented with a management by objectives that constitutes a form of conduct and organization of the government to base its conception in anticipating and preparing early achievements and the demarcation and allocation of responsibilities. So that decision making is a tool to make the best decisions on behalf of the company, affecting management, identifying the objectives that will lead to achieving competitive advantage and market success. The problem of this research focuses on the correlation of the decision-making and management by objectives in the Peruvian company; looking to strengthen political commitment and accountability for results that complements the paradigm of management rules and procedures prevailing in the dosage forms of the Peruvian company.
\end{abstract}

Keywords: Decision making, management by objectives, company.

\footnotetext{
* Doctor en Administración, Magíster en Administración, Magíster en Desarrollo Económico Social, Licenciado en Administración de Empresas Cooperativas, Ex Director del Instituto de Investigación y Ex Decano de la Facultad de Ciencias Administrativas de la Universidad Nacional Mayor de San Marcos. Autor de libros de su especialidad y docente de Pre y Posgrado en universidades públicas.
} 


\section{INTRODUCCIÓN}

Como parte del desarrollo de nuestro país, hemos notado el aporte de las inversiones extranjeras y nacionales con la generación de puestos de trabajo; sin embargo, no debemos desmerecer el aporte de las empresas peruanas que permiten la dinamización de nuestra economía, estas empresas en su mayoría se constituyen por micro, pequeñas y medianas.

A nivel de la organización, se presentan diversos escenarios que merecen ser analizados y ejecutar acciones para la permanencia de la empresa en el mercado. Por eso el gerente y los colaboradores deben estar preparados para enfrentar las situaciones positivas o adversas.

En el presente estudio se analiza la importancia y beneficios de la toma de decisiones y la gestión por objetivos en la empresa peruana para dar alcances a los interesados en la investigación.

\section{PLANTEAMIENTO DEL PROBLEMA}

En los últimos años, el país ha visto el incremento de las empresas peruanas mediante la creación de micro, pequeñas y medianas. Sin embargo, es común ver que así como aparecen también se extinguen y la razón se puede hallar en las trabas que impone la burocracia en nuestro país.

Pero esta no es la única causa del rápido declive, sino también el desconocimiento de la administración de estas pequeñas organizaciones lo que impide su óptimo desarrollo. Es esta última causa la que se analizará en este estudio.

\section{OBJETIVOS}

\section{OBJETIVO GENERAL}

- Establecer la relación de la toma de decisiones y la gestión por objetivos en la empresa peruana.

\section{OBJETIVOS ESPECÍFICOS}

- Analizar las etapas en el proceso de toma de decisiones en la empresa.

- Determinar las características más importantes que deben afianzarse en la gestión por objetivos en la empresa.

\section{JUSTIFICACIÓN}

La principal ventaja competitiva de las organizaciones exitosas reside en la calidad de su gestión, por lo tanto la toma de decisiones es un pilar fundamental para que la gestión por objetivos logre el éxito de la organización. Esto conlleva un posicionamiento en el mercado, aumentando su eficacia competitiva y el valor que perciben los clientes, proveedores, etc., lográndose el efecto boomerang hacia la empresa misma y, por ende, a la sociedad.

\section{HIPÓTESIS}

La toma de decisiones es un factor determinante e influyente en la gestión por objetivos en la empresa peruana, brindándole innovación, competitividad y éxito.

\section{METAS ESPECÍFICAS}

- Que se afiancen las competencias del gestor en la toma de decisiones, para determinar una conducta adecuada para resolver una situación problemática.

- Que la gestión por objetivos cimiente una política de compromisos y responsabilidades por resultados que complemente la gestión para lograr ventajas competitivas.

\section{MARCO TEÓRICO DEL TRABAJO}

\section{EMPRESA}

Según el enfoque tradicional, se define a la empresa como el conjunto de factores de producción coordinados, cuya función es producir y su finalidad viene determinada por el sistema de organización económica en el que la empresa se halle inmersa.

En el sistema capitalista o de economía de mercado, el fin de la empresa ha consistido tradicionalmente en la obtención del máximo beneficio o lucro, mientras que en las economías colectivistas con dirección centralizada el fin de la empresa ha consistido en cumplir los objetivos asignados en un plano más general, de ámbito nacional, regional o local. En definitiva, una empresa es una unidad económico-social integrada por elementos humanos, materiales y técnicos que tiene el objetivo de obtener utilidades a través de su participación en el mercado de bienes y servicios.

\section{CLASIFICACIÓN DE LA EMPRESA}

Las empresas se pueden clasificar según la actividad económica que desarrollan: 
- Empresas del sector primario, que obtienen los recursos a partir de la naturaleza, como las agrícolas, pesqueras o ganaderas.

- Empresas del sector secundario, dedicadas a la transformación de bienes, como las industriales y de la construcción.

- Empresas del sector terciario, que se dedican a la oferta de servicios o al comercio.

Según su constitución jurídica son:

- Empresas individuales, que pertenecen a una sola persona.

- Empresas societarias, conformadas por varias personas. Entre estas tenemos anónimas, de responsabilidad limitada y de economía social (cooperativas), entre otras.

Finalmente, según la titularidad se encuentran:

- Empresas privadas, cuyo capital está en mano de particulares.

- Empresas públicas, controladas por el Estado.

- Empresas mixtas, donde el capital es compartido por particulares y el Estado.

- Empresas de autogestión, el capital es de los trabajadores.

\section{TOMA DE DECISIONES}

La toma de decisiones es el proceso mediante el cual se realiza una elección entre las opciones o formas para resolver diferentes situaciones de la vida en diferentes contextos y consiste en elegir una opción entre las disponibles, a efectos de resolver un problema actual o potencial (aun cuando no se evidencie un conflicto latente).

Los aspectos anteriores toman como base principal la información. Esta información es utilizada para llevar a cabo acciones que beneficien la operación diaria dentro de las organizaciones. Para tomar una decisión, cualquiera que sea su naturaleza, es necesario conocer, comprender, analizar un problema, para así poder darle solución, además requiere de elementos de índole cualitativa y cuantitativa.

En algunos casos, por ser tan simples y cotidianos, este proceso se realiza de forma implícita y se soluciona muy rápidamente, pero existen otros casos en los cuales las consecuencias de una mala o buena elección pueden tener repercusiones en la vida y, si es en un contexto laboral, en el éxito o fracaso de la organización, Por ello, es necesario realizar un proceso más estructurado que puede dar más seguridad e información para resolver el problema. No obstante, la toma de decisiones depende, en gran parte, del estilo de liderazgo de quien las toma. En otras palabras, las acciones que se llevarán a cabo están sujetas a la subjetividad de los individuos que deciden qué hacer con la información.

\section{PROCESO DE TOMA DE DECISIONES}

La separación del proceso en etapas puede ser tan resumida o tan extensa como se desee, pero podemos identificar principalmente las siguientes etapas:

\section{Identificar y analizar el problema}

Esta etapa consiste en comprender la condición del momento y visualizar la condición deseada, es decir, encontrar el problema y reconocer que se debe tomar una decisión para llegar a la solución de este. El problema puede ser actual, porque existe una brecha entre la condición presente real y la deseada, o potencial, porque se estima que dicha brecha existirá en el futuro.

\section{Identificar los criterios de decisión y ponderarlos}

Consiste en identificar aquellos aspectos que son relevantes al momento de tomar la decisión, es decir, aquellas pautas de las cuales depende la decisión que se tome. La ponderación es asignar un valor relativo a la importancia que tiene cada criterio en la decisión que se tome, ya que todos son importantes pero no de igual forma.

\section{Definir la prioridad para atender el problema}

La definición de la prioridad se basa en el impacto y en la urgencia que se tiene para atender y resolver el problema. Esto es, el impacto describe el potencial al cual se encuentra vulnerable y la urgencia muestra el tiempo disponible que se cuenta para evitar o, al menos, reducir este impacto.

\section{Generar las opciones de solución}

Consiste en desarrollar distintas posibles soluciones al problema. Si bien no resulta factible en la mayoría de los casos conocer todos los caminos que se pueden tomar para solucionar el problema, cuantas más opciones se tengan va a ser mucho más probable encontrar una que resulte satisfactoria. 


\section{Evaluar las opciones}

Consiste en hacer un estudio detallado de cada una de las posibles soluciones que se generaron para el problema, es decir, mirar sus ventajas y desventajas, de forma individual con respecto a los criterios de decisión, y una con respecto a la otra, asignándoles un valor ponderado.

\section{Elección de la mejor opción}

En este paso se escoge la opción que, según la evaluación, va a obtener mejores resultados para el problema. Existen técnicas (por ejemplo, análisis jerárquico de la decisión) que nos ayudan a valorar múltiples criterios.

Los siguientes términos pueden ayudar a tomar la decisión, según el resultado que se busque:

Maximizar: Tomar la mejor decisión posible.

Satisfacer: Elegir la primera opción que sea mínimamente aceptable y satisfaga de esta forma una meta u objetivo buscado.

Optimizar: La que genere el mejor equilibrio posible entre distintas metas.

\section{Aplicación de la decisión}

Poner en marcha la decisión tomada para así poder evaluar si la decisión fue o no acertada. La implementación probablemente derive en la toma de nuevas decisiones de menor importancia.

\section{Evaluación de los resultados}

Después de poner en marcha la decisión, es necesario evaluar si se solucionó o no el problema. Si el resultado no es el que se esperaba, se debe evaluar si es porque debe darse un poco más de tiempo para obtener los resultados o si definitivamente la decisión no fue la acertada. En este caso, se debe iniciar el proceso de nuevo para hallar una nueva decisión.

El nuevo proceso que se inicie en caso de que la solución haya sido errónea contará con más información y se tendrá conocimiento de los errores cometidos en el primer intento.

Importancia de la toma de decisiones en grupo

Si bien el supervisor casi siempre toma las decisiones solo, hay ocasiones en que debe aprovechar la ventaja de contar con su grupo de subordinados para hacerlo.

La toma de decisiones en las organizaciones modernas son realizadas en grupo o comités de trabajo, quedan individualizadas en el momento en que ellas pasan a formar parte de las bien estructuradas o estándar. Estas decisiones individuales o grupales tienen cada una de ellas sus ventajas y desventajas, que influyen de manera determinante en el rol de la gerencia de nuestras organizaciones.

Vamos a analizar las ventajas y desventajas del trabajo en grupo o comités:

\section{Ventajas:}

- Información y conocimiento más completos: Lógicamente un grupo logra recopilar más información, teniendo acceso a más fuentes informativas que un solo individuo, independiente de la educación y de la experiencia de este. Por lo tanto, los grupos pueden ofrecer mayores aportes, tanto en la cantidad como en la diversidad para la toma de decisiones.

- Incrementar la aceptación de una solución o bien la variedad de puntos de vista: Muchas decisiones fracasan después de elegida una opinión, debido a que un sector de gente no la acepta como una solución posible. Cada uno de sus integrantes tiene un punto de vista propio que difiere, en cierta medida, del de los demás; como resultado, la cantidad y los tipos de opciones son mayores que los del individuo que trabaja solo.

La participación en grupo facilita una amplia discusión y una aceptación más participativa. Es posible que haya divergencias en los acuerdos, pero se plantea y permite su discusión para cuando ya sea aceptada, sea un compromiso de todo un conjunto. Es difícil que los asistentes al grupo de discusión ataquen o dificulten una decisión que ellos ayudaron a desarrollar. Las decisiones grupales incrementan la aceptación de la solución final y facilitan su instrumentación.

- Incrementan la legitimidad: Los métodos democráticos son aceptados por todos los componentes de la sociedad. Cuando el proceso es grupal, intervienen todos los aditamentos de los ideales democráticos. Si el tomador de decisiones no consulta a otros antes de tomar una de ellas, el hecho del poder que tiene no lo exime de quedar como una persona autoritaria y arbitraria.

Las decisiones grupales no tienen la varita mágica de la perfección, pero sin lugar a dudas 
son las menos peligrosas y, por lo tanto, las que tienen un menor nivel de error.

- Reducción de los problemas de comunicación: Puesto que el grupo participa en la toma de decisión, todos sus integrantes están conscientes de la situación; por lo general, la puesta en marcha de la solución se realiza sin tropiezos. Las preguntas, las objeciones y los obstáculos a los que normalmente se enfrenta la implantación de una decisión con frecuencia desaparecen cuando esta última es resultado de la participación del grupo.

\section{Desventajas:}

- Requieren mucho tiempo: El reunir al grupo toma su tiempo; pero con una buena organización, las reuniones estarán programadas de antemano en un espacio de tiempo oportuno (varía de acuerdo a la organización y no debe ser menor de dos semanas). El resultado es que los grupos consumen más tiempo en alcanzar una decisión a diferencia de un solo individuo.

- Presiones de aceptación: Si bien se supone que todos los miembros del grupo deben sentirse libre para expresar sus opiniones, sugerencias y recomendaciones, no deja de ser cierto que a veces existe cierta presión para que todo el mundo se reúna y acate el consenso general, llamado con frecuencia "pensamiento grupal". Esta presión puede provocar que el grupo pase por alto un consejo o sugerencia positiva de algunos de los presentes. Se presiona a los inconformes para que se ajusten y adhieran a la opinión de la mayoría.

En los grupos existen presiones sociales. El deseo de los miembros del grupo de ser aceptados, por lo tanto, ser protagonistas puede resultar en un intercambio de pareceres condicionado a deseos de una demostración de un liderazgo. Finalmente, se llegará a un mismo resultado que necesariamente debe ser aceptado por todos para tener validez.

- Responsabilidad ambigua: Los miembros de un grupo tienen que compartir la responsabilidad, por lo tanto la individualidad se diluye, dándole un gran valor a los resultados.

- El compromiso: En ciertas ocasiones el grupo se estanca y se muestra incapaz de llegar a un acuerdo sobre qué soluciones recomendar. Obligados a tomar una decisión, se alienta a los miembros a llegar a un compromiso o a darse por vencidos, aceptando una versión diferente de su solución. Este inconveniente es muy usual cuando el grupo se subdivide en grupos más pequeños, cada uno de los cuales apoya una solución diferente.

Cómo lograr que funcione la toma de decisiones en grupo: La toma de decisiones en grupo puede utilizarse con mucha eficiencia si el supervisor maneja la situación como debe ser. Uno de los factores más importantes consiste en ganarse el apoyo de los miembros del grupo, señalándoles el valor de sus aportes en la solución del problema.

Un segundo enfoque muy útil consiste en dar a cada integrante del grupo elementos específicos en que pensar y trabajar, para que pueda reconocer sus aportes; también crear un entorno donde las personas puedan expresarse abierta y francamente y que estimule tanto los aportes creativos como las discusiones sobre las fallas o los errores en que podría incurrirse. Esto último es de especial importancia para evitar el surgimiento del pensamiento grupal.

La gerencia debe tomar decisiones difíciles $\mathrm{y}$ eso hace imposible hacer felices a todos. Momentos como este agregan nuevas tensiones y demandas a todos en la empresa. La gerencia tiene que tomar decisiones difíciles y medidas poco populares. Esto no es nada fácil ni agradable. Además, eso no demuestra que la gerencia sea vil e insensible. Observar a una gran empresa pasar por una gran transición y cambio es como observar a los participantes de un juego de cartas. Algunos ganan, algunos pierden y otros empatan. Como el repartidor de cartas, la gerencia debe "trabajar para el bien" de la organización, asumiendo que, en el proceso, algunas personas serán más golpeadas que otras.

Si usted fuera la persona a cargo, enfrentaría el mismo dilema. Es fácil sentarse a criticar la manera en que la alta gerencia hace las cosas. También es fácil acusar a la gerencia de no preocuparse por la gente. Cuando a uno no le gusta lo que está sucediendo, la tendencia natural es buscar a alguien a quien culpar.

Pero en lugar de señalar a sus superiores, considere la posibilidad de que ellos solo están haciendo lo que deben hacer. Es muy común preocuparse profundamente por los demás, y aún así, no poderles dar todo lo que quieren. 
"Manejar una compañía es fácil cuando no se sabe cómo, pero muy difícil cuando se sabe".

Price Pritchett

\section{GESTIÓN POR OBJETIVOS}

Es una técnica participativa de la planeación y evaluación, a través de la cual los jefes y los colaboradores, conjuntamente, definen aspectos prioritarios, establecen objetivos (resultados) a ser alcanzados en un determinado periodo de tiempo y en términos cuantitativos, dimensionando las respectivas contribuciones (metas), además hacen seguimientos sistemáticamente del desempeño (control) procedimientos a las correcciones necesarias.

La administración por objetivos, también conocida como APO, es una forma de administrar una empresa que consiste en que los subordinados, de forma conjunta con la dirección, deciden los objetivos que se van a perseguir en determinada línea de producción, unidad administrativa, sucursal, etc.

Estos objetivos se constituirán en medidas de desempeño que se evaluarán de forma periódica. Dicha evaluación representará la toma de medidas correctivas, así como también otorgará recompensas con base en los logros conseguidos.

Algo realmente importante de la administración por objetivos es que se parte de objetivos generales que se van desglosando a través de toda la organización hasta hacerlos específicos para cada sección o departamento e incluso llegando a establecer objetivos personales para cada uno de los involucrados en la empresa. De ahí que la participación de los empleados en la definición de los objetivos es vital para su implicación en el logro de ellos.

La gestión por objetivos se basa en la definición de objetivos de cada empleado para después comparar y encaminarlos hacia la mejora de los objetivos de la empresa. Se trata de mejorarlos a través de alinear los objetivos personales con los objetivos generales. Lo ideal es que los empleados reciban apoyo para identificar sus objetivos, plazos para lograrlos y seguimiento continuo de los procesos para obtener retroalimentación que ayude a alcanzarlos.

La gestión por objetivos fue definida por Drucker en 1954, en su libro "La gerencia de empresas”. Según Drucker, los gerentes deben evitar la trampa de la actividad, que los atrapa en las actividades cotidianas y los hace olvidar su propósito: alcanzar los objetivos principales. Uno de los conceptos de la gestión por objetivos establece que todos los gerentes de la empresa, no solo alta dirección, deben participar en el proceso de la planificación estratégica para complementar y mejorar el análisis y las decisiones finales.

Los principios de la gestión por objetivos son:

- Conexión en cascada de las metas y objetivos de la empresa.

- Objetivos específicos para cada miembro de la empresa.

- Toma de decisiones participada por todos los miembros.

- Plazos explícitos de aplicación

- Evaluación sistemática del desempeño.

La gestión por objetivos también introdujo un concepto para comprobar la validez de estos, "SMART", según el cual los objetivos deben ser:

- Específicos (Specific)

- Medibles (Measurable)

- Factibles (Achievable)

- Realistas (Realistic)

- Con un plazo de ejecución (Time-related).

\section{MÉTODO}

La investigación es explicativa, no experimental. El universo son las empresas micro, pequeñas y medianas de Lima Metropolitana, con un total de 40 seleccionadas.

Sin embargo, tal como se explicó líneas arriba ,solo se ha podido trabajar la presente investigación según el juicio de expertos de información, publicada en libros de administración y tesis como Peter Drucker "La gerencia de empresas", Borea, R. Fabián (2004) "La toma de decisiones. Un modelo de análisis integrador", "La toma de decisiones: teorías, modelos y metodología” y Hernández y Rodríguez, Sergio "Introducción a la administración”, entre los más destacados.

\section{RESULTADOS}

La toma de decisiones es un proceso que permite elegir entre distintas opciones en diversos contextos para la resolución de problemas, que dependerá del estilo de liderazgo del ente decisor. 
Por esta razón, es conveniente evaluar todas las variables que incidan en el problema para disminuir riesgos en la organización.

La gestión por objetivos o administración por objetivos (APO) constituye una técnica participativa que permitirá establecer objetivos para cada colaborador o área a la que pertenezcan al observar las funciones que desempeñan y, de esta manera, establecer un periodo de tiempo para que puedan alcanzarlos.

\section{DISCUSIÓN}

La investigación sobre la toma de decisiones y la gestión por objetivos en la empresa peruana es explicativa, ya que buscamos conocer los factores causales que explican nuestro problema de investigación.

El diseño básico de esta investigación es no experimental, debido a que no se harán pruebas, es decir, en esta investigación las variables no van a ser manipuladas. Igualmente es transeccional, porque nuestro objeto de estudio va a ser analizado en un periodo determinado de tiempo. Asimismo, es explicativo correlacional.

\section{CONCLUSIONES}

- Se debe aplicar el proceso de toma de decisiones en las empresas micro, pequeñas y medianas mediante la identificación y análisis del problema, identificación de criterios de decisión y ponderación, definición de prioridades, generación de opciones de solución y evaluación de las mismas, elección, aplicación de la decisión y evaluación de resultados.

- La gestión a través de objetivos designados para cada área y colaborador de la organización, de modo que cada uno aporte de manera significativa a la permanencia y existencia de la empresa.

\section{REFERENCIAS BIBLIOGRÁFICAS}

Bonini, Charles; Asuman, Warren; Bierman, Harold (1999). Análisis cuantitativos para los negocios, Irwin Mc Graw-Hill.
Borea, R. Fabián (2004). "La toma de decisiones. Un modelo de análisis integrador", para el seminario introductorio "La toma de decisiones: teorías, modelos y metodología" de la maestría en Desarrollo Económico Local.

Covey, R. Stephen (1998). Los siete hábitos de la gente altamente efectiva. CoveyLeadership Center.

Chiavenato, Idalberto (2006). Introducción a la teoría general de la administración. Ed. Mc Graw Hill.

Davis, Duan (2001). Investigación en administración para la toma de decisiones, Thomson Editores.

De Bono, Edward (1987). Los sombreros para pensar. Editorial Paidos.

Drucker, Peter (1994). La gerencia de empresas, Editorial Sudamericana.

Fischer, Robert (1999). "El arte de negociar sin saber".

Heller, Robert (1998) La toma de decisiones. Biblioteca Esencial del Ejecutivo.

Hernández, Fernández y Baptista (2010) Metodología de la Investigación. México, Mc Graw Hill.

Hernández y Rodríguez, Sergio (2002) Introducción a la administración. Mc Graw Hill.

Johnson, Spencer (2003) "Sí o no”. Guía práctica para tomar mejores decisiones.

Moody, P. (1992). Toma de decisiones gerenciales, México. Mc Graw Hill.

Mejía, Elías (2005). Metodología de la investigación científica, Lima, Centro de Producción Editorial de la UNMSM.

Reyes Ponce, Agustín (2006) Administración de empresas 2da Parte, Ed. Fca.

Simon, Herbert A. (1994). El comportamiento administrativo. Estudio de los procesos decisorios en la organización administrativa, Aguilar Ediciones. 
